\title{
DESAFÍOS BIOÉTICOS DEL DSM-5: ¿RUPTURA, RECALIBRACIÓN O MÁS DE LO MISMO?
}

Gustavo Figueroa ${ }^{1}$

Resumen: 1] El DSM-5 representa la incorporación de la psiquiatría a la medicina basada en la evidencia. 2] La psiquiatría, propia de la intención, se transformó en la fundamentada en la validación empírica. 3] El DSM-5 es expresión de la ciencia moderna que da prioridad al saber calculador, objetivador, clasificador y operacional. 4] A la ética de la beneficencia, propia de la intención, se superpuso una ética de la autonomía y de la equidad, características de la validación. 5] El DSM-5 no puede determinar si los trastornos psiquiátricos son entidades naturales, tipos prácticos hechos por los investigadores, entes construidos socialmente, rótulos que se agrupan formando un sistema.

Palabras clave: DSM-5, ética, validación, trastornos mentales, medicina basada en la evidencia

\section{Bioethical challenges of the DSM-5. Rupture, recalibration, or more of the same?}

Abstract: 1] The DSM-5 represents the incorporation of psychiatry into evidence-based medicine. 2] The psychiatry proper to the intention was transformed into that based on empirical validation. 3] The DSM-5 is an expression of modern science that gives priority to calculating, objectifying, classifying, operational knowledge. 4] An ethic of autonomy and fairness characteristic of verification was superimposed on the ethics of beneficence proper to the intention. 5] DSM-5 cannot determine whether psychiatric disorders are natural entities, practical types made by researchers, socially constructed entities, labels that are grouped into a system.

Keywords: DSM-5, ethics, validation, mental disorders, evidence-based medicine

\section{Desafios bioéticos do DSM-5: Ruptura, recalibraçáo ou mais do mesmo?}

Resumo: 1] O DSM-5 representa a incorporação da psiquiatria à medicina baseada em evidencia. 2] A psiquiatria, própria da intenção, se transformou na fundamentada em validação empírica. 3] O DSM-5 é expressão da ciência moderna que dá prioridade ao saber calculador, objetivador, classificador e operacional. 4] À ética da beneficência, própria da intenção, se superpôs uma ética da autonomia e da equidade, características da validação. 5] O DSM-5 não pode determinar se os transtornos psiquiátricos são entidades naturais, tipos práticos criados pelos investigadores, entes construídos socialmente, rótulos que se agrupam formando um sistema.

Palavras chave: DSM-5, ética, validação, transtornos mentais, medicina baseada em evidencia

\footnotetext{
${ }^{1}$ Departamento de Psiquiatría, Escuela de Medicina, Universidad de Valparaíso, Chile Correspondencia: gfigueroacave@gmail.com
} 
En el año 2013 aparece el DSM-5 (Diagnostic and Statistical Manual of Mental Disorders. Fith Edition) de la Asociación Psiquiátrica Americana(1), que constituye la culminación del proceso de diagnosticar en psiquiatría — qué se entiende por trastorno mental-, diagnosticar que se remonta al nacimiento de la medicina como tékhnê iatriké(2). El concepto original griego para diagnosticar era diagigôskein, que admitía un doble sentido: por un lado, discernir, distinguir, y por otro conocer atravesando o recorriendo, pero profundizando, penetrando en lo que no se ve desde lo que en ella es aparente. Por otro ángulo, la enfermedad recibió desde temprano distintos nombres: en griego, nósos (daño), páthos (padecimiento, dolencia), arrostía (desvalimiento), asthéneia (debilidad) y en latín, morbus (lo que hace morir), passio (padecimiento), infirmitas (falta de firmeza) (3), aunque Aristóteles pone como excepción la manía, que se identifica con "la posesión divina" cuando proviene de "un temperamento natural" (4). Ambos sentidos - diagnóstico y enfermedad psiquiátricos — han experimentado una transformación sustancial hasta la actualidad, en parte consecuencia de las diferentes experiencias éticas que los ha hecho posibles y sustentados(5).

El presente trabajo consta de tres partes: desarrollo de los fundamentos del DSM-5; ética de la ciencia psiquiátrica inherente al proceso de diagnosticar y el enfermar psiquiátrico como modo de existencia.

\section{El DSM-5: culminación del proceso diagnósti- co en la psiquiatría}

1. Antecedentes. Posterior al término de la Segunda Guerra Mundial, diagnosticar en psiquiatría entró en profundo descrédito: etiqueta que representa un estigma denigratorio, inutilidad para efectuar las (psico) terapias rigiéndose según la denominación nosológica, caos que impide comparar los resultados entre diferentes estudios y centros clínicos, y ausencia de guías específicas para el empleo de psicofármacos recientemente introducidos en la psiquiatría.

2. La conmoción epistemológica. La insatisfacción profesional ante el estado defectuoso de la psiquiatría se transmutó en un cambio de sus fundamentos en una triple dimensión. Por una parte, la psiquiatría práctica que estaba basada en la "intención" — deseos, experiencia, probabilidades subjetivas - cambió por una sustentada en la "validación" —verificación, experimentación-; por otra, un modelo cimentado en las ciencias sociales — "modelo biopsicosocial" - mudó en un modelo médico - "modelo basado en la evidencia”-(6). Por último y de importancia para el presente estudio, una ética con un acento puesto en la beneficencia propugnó un proyecto de otra ética esta vez centrada en la autonomía.

\section{Pasos hacia una psiquiatría basada en la eviden-} cia. El RDC (Reseach Diagnostic Criteria) de 1978 fue el resultado final de un grupo de investigadores de la Universidad de Washington, destinado a determinar la evidencia empírica de cinco trastornos psiquiátricos usando validadores empíricos: descripción clínica precisa, estudios de laboratorio, delimitación específica entre los trastornos, análisis de seguimiento y estudios familiares(7). Los fundamentos, la metodología y la evidencia obtenidos fueron decisivos para iniciar la confección del DSM-III (1980), DSM-III-R (1987), DSM-IV (1994), DSM-IV-TR (2000) y DSM-5 (2013), representando cada manual una reorientación de las bases, concepciones más específicas e investigaciones más estrictas.

4. El DSM-5 entre la validación y la experimentación. La caracterización final de trastorno mental, expresión de las investigaciones de la literatura y de estudios sistemáticos de campo, fue "es un síndrome caracterizado por una alteración (disturbance) clínicamente significativa en la cognición, regulación emocional o conducta de un individuo, que refleja una disfunción en los procesos psicológicos, biológicos o del desarrollo que están a la base del funcionamiento mental. Los trastornos mentales habitualmente están asociados con malestar (distress) o discapacidad significativa en actividades sociales, ocupacionales u otras importantes. Una respuesta esperable o culturalmente aprobada a un estresor o pérdida común, tal como la muerte de alguien querido, no es un trastorno mental. Conductas socialmente desviadas (ej. políticas, religiosas o sexuales) y conflictos que primariamente son entre un individuo y la sociedad, no son trastornos mentales, a menos que la desviación o conflicto resulte de una disfunción en el individuo, como se describió más arriba". 
La definición fue resultado de aumentar a 11 los validadores de factores de riesgo: genéticos, familiares, ambientales específicos compartidos, sustratos neurales compartidos, marcadores biológicos compartidos y antecedentes temperamentales compartidos; y validadores de cuadros clínicos: anormalidades compartidas de procesamientos cognitivos y emocionales, similitud de síntomas, altas tasas de comorbilidad, curso de la enfermedad y respuesta al tratamiento.

5. El DSM-5 y el RDoC. Producto del trabajo desde 1999 a 2012 fue la elaboración de un manual subdividido. El DSM-5, que describe 11 categorías diagnósticas clínicas en sus diferentes niveles: criterios, subtipos, especificadores, severidad, curso, prevalencia e inespecíficos. Como anexo se agregó un grupo especial de ocho trastornos como "condiciones para estudios posteriores”, señalando que la investigación aún no puede afirmar su realidad como tipos diagnósticos, pero que representan una prioridad futura por su trascendencia clínica y conceptual. Por otra parte, ganancia del estudio representó la necesidad de una pronta confección del RDoC (Research Domain Criteria), un manual destinado con exclusividad a la investigación basada en la validación y verificación, complementario pero independiente del $\operatorname{DSM}-5(8,9)$.

6. El DSM-5 y el ICD-11. La psiquiatría basada en la evidencia, propia del DSM-5, cuyo objetivo es que sus resultados sean positivos y ciertos, proyecta que sea generalizable a toda la población actual según se la entiende en estos tiempos; de ahí su adición de códigos compatibles con la clasificación diagnóstica ICD-11 de la Organización Mundial de la Salud, que está, a pesar de sus numerosas prórrogas en relación a la psiquiatría, finalizando su redacción definitiva (International Statistical Classification of Diseases and Related Heath Problems)(10).

\section{DSM-5: ética del diagnosticar en psiquiatría}

Pasar de una psiquiatría práctica basada en la "intención" a una psiquiatría científica sustentada en la "validación" significa una modificación de los fundamentos bioéticos en la base. La psiquiatría de la intención supone que esta intención es doble: intención clínica de beneficencia del psiquia- tra e, indirectamente, intención cognoscitiva por el principio del doble efecto -investigación que solo de manera colateral o casual se ejecuta a propósito de terapias empleadas en clínica-; esto es, nada que no sea clínico primariamente se puede justificar éticamente, incluidos los conocimientos adquiridos, por importantes que ellos sean. Contrariamente, la psiquiatría basada en la evidencia significa que nada que aspire a ser clínico se puede aseverar si no es validado con anterioridad, en seres humanos de preferencia, y no de manera casual o colateral sino controlada. Vale decir, se pasa de una validación "por accidente" a una validación "propiamente tal". Sin embargo, transitar de experimentos "casuales" a experimentos "controlados" implica ir más allá de una ética de beneficencia: es adoptar una ética autónoma, porque los ensayos pueden ser no solamente no beneficiosos para el paciente, sino aun riesgosos o perjudiciales. En otras palabras, el respeto a la autonomía del sujeto significa ascender desde la ética basada en la beneficencia a la ética sustentada en la autonomía — consentimiento informado-, conocimiento explícito, suficiente y adecuado del paciente a todas las circunstancias del proceso de validación(11). Pero no basta. Para llevar a cabo el estudio, la selección de pacientes requiere considerar necesariamente el principio de justicia, para ejecutarlo equitativamente. En otras palabras, la "intención" subjetiva se trasmutó éticamente en la "evidencia" de las pruebas objetivas: consentimiento informado, ponderación beneficio/riesgos y selección equitativa de muestras.

El DSM-5, incluido el RDoC, significó transitar de clínico a investigador, de lo observacional a lo experimental, de una perspectiva retrospectiva a una prospectiva, de pacientes según sus necesidades a grupo control aleatorizado, de conocer lo indicado a ignorar lo prescrito, de opinar sobre los resultados a medir con exactitud el desenlace, de la analogía a la evidencia. Mientras la ponderación riesgo/beneficio satisface la ética de la beneficencia, el consentimiento informado satisface la de autonomía y la selección equitativa de sujetos la de justicia.

Aristóteles acentuó que existían distintos tipos de saberes o de estar en la verdad: propias del intelecto teórico, inteligencia o noûs, ciencia o episteme y sabiduría o sophia; y propias del intelecto práctico, 
prudencia o phrónesis y arte o técnica o téhkné, y además experiencia o empeiría(12). El DSM-5, producto de sus metas anheladas durante cerca de veinte años de confección, no pretende ser ciencia entendida como lo hacían los griegos, esto es episteme - saber cierto, universal, deductivo- sino un saber práctico o dóxa - particular, probable, proporcionado-, por más que su intención sea ceñirse lo más estrictamente a las leyes de la ciencia positiva(13). El saber propio de la medicina, y por tanto el saber de la psiquiatría, ha variado en la historia y por esto ha mutado la ética que lo ha sustentado. En sus inicios griegos fue una ética de la virtud, en la modernidad una ética de los derechos y deberes, y actualmente, el tiempo del DSM-5, tiende progresivamente a una ética de la responsabilidad, al tener en cuenta el contexto, tanto histórico como social y cultural, pero también individual — con todo y con todos(14), aunque procurando tener siempre presente la advertencia de Aristóteles, "podría ser de otra manera", fragilidad inherente al objeto mismo de la ética(15-17).

Empero, MacIntyre ha hecho notar que estamos en una sociedad con múltiples éticas, que se disputan la primacía valorativa apelando a la coherencia, plausibilidad y prácticas sociales de sus propuestas, por lo que estamos "después" (after) de la virtud médica original. Esta situación hace necesario ahora ir "hacia" (after) la virtud nuevamente, ya que es a la moralidad intrínseca o inherente al ejercicio médico en sí mismo y no a los usos sociales contingentes, modificados o acomodadizos con los tiempos, lo que implica que la psiquiatría precisa volver a empuñar sus fundamentos éticos primarios como tékhnê iatriké(18). Sin embargo, conviene tener en cuenta que "virtud" proviene de la palabra latina virtus, que significaba varón y no fue una buena traducción de la original griega areté, que a su vez tenía un sentido primariamente físico antes que moral; pero, de cualquier manera, corresponde más exactamente a excelencia: el DSM-5, entendido como psiquiatría en cuanto téhkné iatriké, necesita nuevamente estar movido por la areté o excelencia. En otros términos, el problema es el de la moralidad esencial a la psiquiatría, su fin intrínseco(19).

Ortega y Gasset señaló que la medicina [psiquiatría] se ha convertido en ciencia moderna porque su "modo de pensar" se ha diferenciado del de la ciencia antigua y, por ello, revela una realidad diferente, que va mucho más allá de si la actual es exacta y la anterior era imprecisa. Mientras la griega averiguaba qué son "las cosas", cuál es el ser de las cosas [desde sí mismas], la ciencia moderna se afana por saber dónde, cuándo y cómo se presentan "los fenómenos" — es el procedimiento de sustituir "las cosas" como son en sí mismas por "fenómenos" que, aunque tengan poco que ver con la realidad de la cual hablan, son más precisos, la llamada precisión objetiva: un "por qué" que siempre es un "cómo" que recae sobre un "quién" $(20,21)$.

Heidegger está de acuerdo en que la psiquiatría es ciencia moderna, lo que implica que se distingue de la ciencia antigua por un doble aspecto: "investigación" y "teoría de la realidad". Por un lado es "investigación" (Forschung) y su particularidad no está solo en el método sino, ante todo, en demarcar por adelantado "un" campo de conocimiento - "la vida psíquica en sus manifestaciones patológicas y, por ello también, simultáneamente, sanas-. Ella concibe esto desde la objetividad de la unidad corporal-espiritual-psíquica del hombre entero. En la objetividad de la psiquiatría... la existencia-humana (Dasein) en donde ex-siste el hombre en cuanto hombre, queda como lo inabarcable para la psiquiatría" (22). Vale decir, revela un ámbito circunscrito, delimitado y especificado con precisión, aunque anticipadamente; como tal, es un esquema, una perspectiva de la realidad que pre-determina tanto el método como el conocimiento. La estricta sujeción a ese proyecto-previo (Vorentwurf), esto es, aprehender que siempre encuentra solo lo que "su" tipo de representación ha admitido con anterioridad como objeto posible, es su pre-supuesto fundante, instaura el rigor de su investigación y es mathémata, aunque entendido en un sentido griego: todo lo que el psiquiatra puede conocer a priori en su objeto, en lo psíquico el psiquismo y en su cuerpo la corporeidad. Tal proceder sistemático desemboca en que "lo conseguido en el hecho de tal hacer es lo efectivo (wirklich), lo cierto y lo seguro (lo que existe de hecho)"(23). Vale decir, "la ciencia [psiquiátrica] concibe gracias a ello algo real, de acuerdo con lo cual se rige objetivamente". Cabe la pregunta: jes el paciente psiquiátrico eso real? Heidegger res- 
ponde de manera clara que no: "la ciencia [psiquiátrica] atañe solamente a lo que "su" modo de concebir ha admitido previamente como posible objeto para ella" (23).

Por otro lado, el DSM-5, en tanto psiquiatría vigente actual, es asimismo ciencia moderna diferente de la antigua, porque es "teoría de la realidad"; y esto en su doble aspecto, de teoría y de realidad. "La realidad" (Wirklichkeit) proviene de hacer o efectuar (wirken). Lo real y actual es lo que hace acto de presencia, lo hecho presente (An-wesen) resultado de una operación o de un acto —en el fondo, lo ex-traído por una acción humana o lo pro-vocado por la ejecución de un sujeto- Con otra expresión, la realidad que se le adjudica a lo psíquico es un resultado (Er-gebnis), un efecto (Er-folg), lo obtenido (das Erwirkte) como producto (das Herausgestellte) de un trabajo (leisten) de un psiquiatra que lo consigue por su acto de investigar(24). Además, el DSM-5 es "teoría” y, en tanto tal, contemplación (Betrachtung), expresión que proviene del latín tractare, obrar, elaborar. Tratar algo, elaborar, significa ocuparse de algo, perseguirlo, asaltarlo, apremiarlo, ajustarlo para tenerlo seguro. "Según esto, la teoría [psiquiátrica] como Betrachtung es el reelaborar ajustador y asegurador de lo real [psíquico]". La frase voluntariosa del Premio Nobel Max Planck de que "es real lo que se deja medir", dictamina, decide, decreta y afirma qué debe valer en la ciencia, "el proceder ejecutivo-asegurador", en el fondo, es un calcular (berechnen); calcular no es solo operar con números, sino "contar con algo", hacerlo entrar, adecuarse y ajustarse a los planes y designios del investigador.

Heidegger y Ortega y Gasset, al señalar que la ciencia moderna es un nuevo modo de pensar, significan que es un pensar que da prioridad a la actitud instrumental, clasificatoria, operacional, calculadora, objetivadora. La objetividad conseguida consiste en acercarse a lo mental para apresarlo, cogerlo y disponer de él mediante la representación. La representación (Vorstellung) es un traer ante sí (vor-stellen) lo que se presenta, y lo puesto-delante, en-frente (Gegen-stehen) es la respuesta que el investigador del DSM-5 arroja al reto - es lo ob-jetivo (lo que se lanza) para dominar y domar y, en este sentido, unificar-. Pero en la representación el primer representado, fundamento de toda certeza, es el sujeto. Por eso, aunque solo por eso, pasa el investigador a ocupar el puesto de ente privilegiado que ostenta la primacía entre todo ente, esto es, entre todo lo cierto, entre todo lo real. La objetividad que de él emana ob-liga (zwingend) a todos, compromete a todos, es válida para todos; nadie ni nada se puede sustraer a la universal objetivación del investigador(25). Heidegger hace presente que la objetividad, conquistada con esmero, sistema y rigor por la ciencia moderna, interiormente encierra una consecuencia, a pesar de que es correcta. La objetividad [conseguida por la psiquiatría] no pasa de ser "una" de las maneras posibles de hacerse presente la realidad, pero no la incondicional y, sobre todo, no la fundamental, sino marginal, y atenerse a ella de forma exclusiva o absoluta, desfiguradora de lo que se muestra, aun aniquiladora (Vernichtung) especialmente cuando se refiere a algo tan sutil como la intimidad del ser humano(26).

El DSM-5 es ciencia moderna, por ello abarca una vertiente científica y una vertiente ética. Como expresión de la ciencia moderna, le es inherente una doble ilusión, que en el fondo es un aniquilamiento del asunto o materia (Sache) que investiga: por una parte, cree alcanzar la realidad con primacía sobre todos los otros modos de experimentar o experienciar; por otra, la apariencia que los trastornos mentales pueden ser igualmente trastornos mentales pese a la investigación científica de la realidad(23). En cuanto proyecto, éticamente el DSM-5 es sostenido y expresa un fundamento moral, un principio producto de una experiencia moral; como toda experiencia y acción humanas morales, el DSM-5 muestra el fracaso, malogro o fractura inherente a ellas, una brecha o vacío moral (the moral gap), quiebre, desfonde o límite que significa que nada que el hombre ejecute, cumple completa y satisfactoriamente todo lo que debe, vale decir, su deber, y así lleva la impronta trágica del deber no cumplido(27).

\section{Enfermar psiquiátrico como modo de existen- cia}

En 1946 la Organización Mundial de la Salud declaró que "la salud es un estado de completo (complete) bienestar físico, mental y social, y no sólo la ausencia de enfermedad (disease) o pade- 
cimiento (infirmity)" (28). Esta declaración parece ser no solo diferente, sino estar en contraposición con lo que el DSM-5 elaborará en los años siguientes.

Hay que observar con cierto cuidado si, detrás de esta diferencia, no hay un igual principio. Ricoeur ha hecho notar lo que los moralistas antiguos y medievales calificaban como concupiscencia essen$d i$, vanagloria de ser, la noción insolente de la salud, el sentimiento de poder de la "buena salud", ese sentimiento de poder, de poder decir puedo esto y aquello, el valor engañoso y auto-engańador de normas definitivas, absolutas: la depreciación irreverente hacia lo patológico, la exclusión de la vulnerabilidad y de lo perecedero(29). Ahí se esconde lo que posteriormente emergerá y será definitorio de la ciencia moderna y con ello de la era técnica contemporánea, es decir, del DSM-5.

Heidegger ha señalado que la modernidad, por tanto la ciencia moderna, des-cubre, de-vela, al estar fundada, tener su fundamento ontológico último, que le permite revelar toda realidad gracias a que descansa en aquello que se puede denominar la acuñación destinadora: Ge-stell, posible de traducir como lo dis-puesto, la im-posición, la posición-total. A este modo de donarse el ser (Sein) actual, moderno y posmoderno, le es inherente una modalidad del des-cubrir: des-ocultar provocante(30). El "completo bienestar" de la definición de la Organización Mundial de la Salud es un dominar, asignar condiciones severas e irrenunciables a lo que el hombre es, tiene o debe ser como un todo, imponiéndole estipulaciones, haciéndole entrar en vereda en el sentido de meterlo en razón. De alguna manera se corresponde en el plano ético a lo que Natorp y Nelson llamaron "ética del éxito" y Weber "ética del poder", beligerante en cuestiones de valor, valorizar solo los métodos propios de la ciencia natural, atenerse valorando exclusivamente a los "hechos" (31); auténtica violencia, porque dictamina sobre algo rigurosamente necesario para el progreso del conocimiento objetivo del mundo de la salud y enfermedad, atenerse con exclusividad a lo que Comte llamó "el régimen de los hechos"(32).

Ricoeur acentúa que caracterizar la enfermedad como algo que falta, deficiencia, defecto, impotencia, es desconocer, negar con furor que, asimis- mo, es portadora de valores positivos y, en el caso del paciente psiquiátrico, está a la base del desprecio hacia los enfermos mismos, de su exclusión en los planos interpersonales, sociales e institucionales. La enfermedad mental, entendida como vulnerabilidad en tanto puro déficit, menoscabo, no se la considera como portadora de valores, digna de estima y de respeto. Contrariamente, puede ser un proyecto existencial de búsqueda indefinida, interminable de reconocimiento de sí mismo en términos de identidad personal, de estima de sí y de reconocimiento por los otros para que estos desplieguen recursos de convivencia, de simpatía, de vivir y de sufrir. "Ser" enfermo mental es otra manera propia y auténtica de ser-en-el-mun$\operatorname{do}(29)$.

Jaspers apunta que lo abarcador (Umgreifende) es el fundamento de la existencia, desde donde se experimentan, en toda su profundidad y trascendencia, las "situaciones-límite" — la enfermedad, el sufrimiento, la muerte, la culpa-, esto es, los fracasos inherentes a la condición humana. Vivir la vida desde el fundamento significa empuñar la amenaza inscrita en cada ser humano, coger las insuficiencias ínsitas en cada sujeto, apresar la falibilidad insoslayable del deber nunca cumplido o "culpa metafísica", y del vivir defectuoso del enfermar, pero que "da el impulso fundamental que mueve a encontrar en el fracaso el camino que lleva al ser"(33-35).

\section{Seamos realistas, pidamos lo imposible}

1. El DSM-5 constituye el proyecto de la incorporación de la psiquiatría a los fundamentos de la medicina del nuevo siglo, progresando de manera sistemática, racional y verificada hacia la medicina basada en la evidencia o sometida a la validación empírica.

2. Expresión de la ciencia moderna, que ha dejado atrás la ciencia propia de la medicina original, el DSM-5 representa un nuevo modo de pensar que, más que centrarse en cómo son en sí mismos los trastornos mentales, da prioridad a una actitud que circunscribe por adelantado "un" aspecto de la realidad de los trastornos: lo objetivable, calculable, clasificable, operacionalizable.

3. El DSM-5 significa transitar de una medicina 
antigua basada en la intención, propia de la ética de la beneficencia, a un moderna, fundamentada en la verificación, cimentada en una ética que, admitiendo la beneficencia, prioriza la autonomía y suscribe simultáneamente una ética propia de la equidad.

4. El fin inherente a la psiquiatría, por ello la moralidad en la base del DSM-5, en una sociedad donde reina la poliaxiología contingente, históri$\mathrm{ca}$, acomodadiza, no permite determinar con precisión ni con certeza: la excelencia, virtud o areté; el deber y los derechos; la responsabilidad.
5. En cualquier caso, el DSM-5 debe aceptar una doble insuficiencia o limitación, ética y epistemológica. Por el lado ético, el ineluctable fracaso moral, quiebre, confín o límite ético de todo su propósito, aspiración, ambición o deseo humano; llámese excelencia, deber o responsabilidad.

6. Por la vertiente epistemológica, los trastornos (disorders) psiquiátricos diagnosticados siguiendo las pautas del DSM-5 son ¿entes esenciales? ¿Entes construidos socialmente? ¿Entes prácticos para uso clínico? ¿Proyectos existenciales de realización personal? ¿Agrupaciones de redes con propiedades mecánicas? ¿Rótulos que se agrupan formando un sistema?(36-39).

\section{Referencias}

1. American Psychiatric Association. Diagnostic and Satistical Manual of Mental Disorders. Fith Edition. DSM-5 ${ }^{\mathrm{TM}}$. Washington: American Psychiatric Publishing; 2013.

2. Laín Entralgo P. El diagnóstico médico. Historia y teoría. Barcelona: Salvat; 1982.

3. Laín Entralgo P. Antropología médica para clínicos. Barcelona: Salvat; 1984.

4. Aristóteles. El hombre de genio y la melancolía. Problema XXX, 1. Barcelona: Sirmio, Quaderns Crema; 1996.

5. Figueroa G. Historia de la psiquiatría. En: Ivanovic-Zuvic F, Correa E, Florenzano R, editores. Texto de psiquiatría. Santiago de Chile: Ediciones de la Sociedad de Neurología, Psiquiatría y Neurocirugía; 2017: 46-61.

6. Greenhalgh T. How to read a paper: The basics of evidence-based medicine and healthcare. $6^{\text {th }}$ edition. Hoboken: John Wiley \& Sons; 2019.

7. Kendler KS, Muñoz RA, Murphy G. The development of the Feighner criteria: a historical perspective. Am J Psychiatry 2010; 167: 134-142.

8. Figueroa G. DSM-5 ¿La incorporación definitiva de la psiquiatría en la medicina? Rev Méd Chile 2019; 147: $475-479$.

9. Insel T, Cuthbert B. Research Domain Criteria (RDoC): toward a new classification framework for research on mental disorders. Am J Psychiatry 2010; 7: 748-750.

10. World Health Organization. ICD-11. International Statistical Classification of Diseases and Related Health Problems. $11^{\text {th }}$ revisión. Volume 1. Geneva: World Health Organization; 2015.

11. Gracia D. Profesión médica, investigación y justicia sanitaria. Santa Fe de Bogotá: El Búho; 1998.

12. Aristóteles. Metafísica. 2 volúmenes. Madrid: Gredos; 1970.

13. Kendler KS. The nature of psychiatric disorders. World Psychiatry 2016; 15: 5-12.

14. Weber M. Politik als Beruf. Wissenschaft als Beruf. Berlin-München: Dunker \& Humblot; 1959.

15. Zubiri X. Cinco lecciones de filosofía. Con un nuevo curso inédito. Madrid: Alianza; 2009.

16. Gracia D. Como arqueros al blanco. Estudios de bioética. Madrid: Triacastela; 2004.

17. Aristóteles. Ética a Nicómaco. Madrid: Centro de Estudios Constitucionales; 1981.

18. MacIntyre A. After virtue. A study in moral theory. $3^{\text {rd }}$ edition. Notre Dame, Ind: University of Notre Dame Press; 2007.

19. Pellegrino E. The internal morality of clinical medicine: a paradigm for the ethics of the helping and healing professions. J Med Philos 2001; 26: 559-579.

20. Ortega y Gasset J. La idea de principio en Leibniz y la evolución de la teoría deductiva. Obras Completas VIII. Madrid: Revista de Occidente; 1962: 59-356.

21. Zubiri X. Naturaleza, Historia, Dios. 9a edición. Madrid: Alianza; 1987.

22. Heidegger, Martin. Wissenschaft und Besinnung. En: Vortäge und Aufsätze. 5. Aufl. Pfullingen: Neske; 1967: $41-66$.

23. Heidegger M. Das Ding. En: Vorträge und Aufsätze. 5. Aufl. Pfullingen: Neske; 1967: 157-175. 
Desafíos bioéticos del DSM-5: ¿̨ruptura, recalibración o más de lo mismo? - Gustavo Figueroa

24. Heidegger M. Die Frage nach der Techknik. En: Vorträge und Aufsätze. 5. Aufl. Pfullingen: Neske; 1967: 9-40.

25. Heidegger M. Die Zeit des Weltbildes. Gesamtausgabe 5. Frankfurt: Klostermann; 1984: 69-104.

26. Heidegger, Martin. Platons Lehre von der Wahrheit. Mit einem Brief über del "Humanismus". 2. Aufl. Bern: Francke; 1954.

27. Hare JE. The moral gap: Kantian ethics, human limits, and god's assistance. Oxford: Clarendon Press; 1996.

28. World Health Organization. Constitution of the World-Health-Organization. Public Health Repo 1946; 61: 1268-1277.

29. Ricoeur P. Le juste. 2. Paris: Éditions Esprit; 2001.

30. Heidegger M. Was Heißt Denken? En: Vorträge und Aufsätze. 5. Aufl. Pfullingen: Neske; 1967: 123-137.

31. Weber M. Die protentantische Ethik und der Geist des Kapitalismus. Stuttgart: Anaconda; 2018.

32. Comte A. Cours de philosophie positive. Vol 1. Paris: Create Space Independent Publishing Platform; 2016.

33. Jaspers K. Philosophie II. Existenzerhellung. 4. Auf. Berlin-Heidelberg, Springer; 2008.

34. Jaspers K. Einführung in die Philosophie. Zürich: Artemis-Verlag; 1949.

35. Figueroa G. La "Psicopatología General" de K Jaspers en la actualidad: Fenomenología, comprensión y los fundamentos del conocimiento psiquiátrico. Rev Chil Neuro-Psiquiat 2000; 38: 167-186.

36. Angst J. Psychiatric diagnoses: the weak component of modern research. World Psychiatry 6; 2: 94-95.

37. Marková IS, Berrios GE. Epistemology of mental symptoms. Psychopathology 2009; 42: 343-349.

38. Kendler KS, Zachar P, Craver C. What kind of things are psychiatric disorders? Psychological Medicine 2011: 41: 11431150 .

39. Zachar P. Psychiatric disorders: natural kinds made by the world or practical kinds made by us? World Psychiatry 2015; 14: $288-290$

40.

Recibido: 7 de diciembre de 2020

Aceptado: 18 de diciembre de 2020 